\title{
LES SUBSTANCES ACTIVES SUR LE COMPORTEMENT A L'INTÉRIEUR DE LA RUCHE
}

\author{
PAR \\ Rémy CHA UVIN \\ Station de Recherches Apicoles, Bures sur Yvette.
}

Ia conception de la ruche comme superorganisme, qui s'impose souvent à l'observateur presque malgré lui, ne s'est guère révélée jusqu'à présent comme " utilisable ": c'est-à-dire qu'elle n'a pas suggéré beaucoup d'expériences. A peine aurait-on osé penser jadis que ce "superorganisme " possédait une circulation très intense, qui évoque en plus d'un endroit celle dont est le siège notre système artériel ou veineux. Mais Nixon et Ribbands (I95I) ont montré qu'il suffisait de distribuer à six abeilles d'un groupe de Io 000 à 15000 un peu de phosphore radioactif pour que $70 \mathrm{p}$. Ioo des membres de la colonie en contiennent 24 heures après. On juge à quel point l'échange de nourriture (activité quasi essentielle des abeilles dans la ruche) doit être actif pour que la répartition du radioisotope se fasse aussi rapidement. Les choses en étaient là lorsque PAIN découvrit que les cadavres de reine inhibaient, aussi bien que la reine vivante, le développement des ovaires des ouvrières; BUTLER prouva à peu près en même temps que le développement des cellules royales était sous la dépendance d'une substance tégumentaire de la reine léchée par les ouvrières.

C'est là l'expérience cruciale qui a marqué le départ des recherches d'hormonologie chez les abeilles. Hormonologie assez particulière, puisque les substances en cause quittent le corps et sont véhiculées d'individu à individu par le canal des échanges nutritifs. C'est pourquoi il vaut mieux les désigner par un nom spécial "ectohormones» ou mienx "phérormones ", comme le proposent BUTENAND't et KarLSON.

En effet, l'expérience des reines mortes oblige à admettre que le stimulus qui provoque les modifications précitées est de nature chimique. On savait déjà, à vrai dire, depuis les recherches de HESS (I942) que si les abeilles peuvent entrer en contact corporel avec la reine, elles gardent 
de petits ovaires; mais si deux grilles espacées d'un centimètre les en séparent, alors les ovaires grossissent : une seule grille par contre permet les contacts et on ne voit pas alors de signe d'orphelinage dans la ruche. Donc quelque chose passe de la reine aux ouvrières qui n'est pas seulement l'odeur (elle traverserait les deux grilles) ; et depuis les expériences de PAIN et BUTLER nous savons que ce "quelque chose ", cette phérormone, se transmet par léchage ou échange de nourriture et qu'elle est très stable (elle existe encore sur des reines conservées en collection depuis trois ans, d'après PAIN).

Ce terme de phérormone, nouveau en biologie de l'insecte, vient d'être parfaitement défini par KARI,SON dans une revue récente (I960). Il s'applique à toutes les substances qui sont émises à l'extérieur d'un individu et reçues par un autre individu de la même espèce, chez lequel elles produisent une réaction spécifique; elles agissent en quantité minime. Nous sommes parfaitement d'accord avec le terme qui doit remplacer celui d'ectohormone, d'abord employé par BETHE (I932) et assez mal chosi. Mais à propos des abeilles tout au moins, il nous paraît déjà nécessaire de fragmenter les phérormones en deux groupes, où se classent des substances dont l'action est opposée, et qu'il ne serait sans doute pas raisonnable de laisser sous la même dénomination ; nous appellerons donc, parmi les phérormones épagines (voir plus loin) les substances de reconnaissance ou de familiarisation, et répulsines celles qui induisent l'alarme, la fuite ou le combat. Comme KARI,son le fait justement remarquer ce serait à tort que l'on classerait, à l'exemple de BETHE, même les $s u b$ stances attractives d'origine végétale parmi les phérormones ; c'est pourquoi je proposerais que le pollen, la propolis ou les nectars, qui attirent vivement les abeilles soient désignés sous le nom d'allectines (voir plus loin); je ne traiterai d'ailleurs pas ici de ce dernier groupe de substances.

\section{Efỉets de la phérormone.}

La phérormone des reines mortes a les mêmes effets que la phérormone des reines vivantes. Dans beaucoup de cas, un extrait de reine peut remplacer un cadavre de reine.

\section{Action sur les cellules royales.}

Test de Butler. - Il consiste à introduire au milieu de 200 ou 300 abeilles conservées à l'étude et orphelines depuis quelques heures un fragment de cadre de couvain frais ; dans ce cas, elles commencent à ébaucher des cellules royales; si on pique alors sur le cadre un cadavre d'ouvrière enduit de miel et sur lequel on a déposé de la phérormone, les abeilles viennent le lécher; alors les cellules royales ne se développent pas (Butler et GibBons, I958). 
Test de Vuillaume. - Il porte sur l'inhibition immédiate des cellules royales, alors qu'on pourrait dire que le test de BUTLER a pour objet l'inhibition médiate: VuildaUne greffe des jeunes larves dans des cupules de verre, que les abeilles d'une ruche orpheline transforment très facilement en cellules royales en les prolongeant d'un tube de cire. S'il badigeonne au préalable les cupules d'une solution très étendue de phérormone, les abeilles abandonnent les cupules, alors que les cupules témoins sont acceptées sans difficulté (Vuthadume, I957).

Degré de spécificité de la réaction d'inhibition des cellules royales. Dans une note très récente (1959) BUTLER, CAL,Low et JohnSTON disent avoir isolé à l'état pur la phérormone de la reine. Pour cela, ils ont extrait des glandes mandibulaires de la reine (nous verrons que c'est là en effet que s'amasse la phérormone) une substance qui s'est révélée après chromatographie comme voisine de l'acide décénoïque isolé par BUteNANDT dans la gelée royale; elle n'en différerait que par un groupe carbonyle non conjugué. Ce corps présenté aux abeilles suivant le test de BUTLER, inhibe la construction des cellules royales. S'ensuit-il pour cela qu'il s'agisse de toute la phérormone? Certainement pas, mais tout au plus d'une de ses composantes. En effet :

a) Nous verrons dans un moment que la phérormone de la reine n'est pas simple, mais que c'est un mélange de plusieurs corps : au moins deux (si ce n'est trois), l'un inhibiteur des cellules royales mais non attractif pour les abeilles; 1'autre certainement différent du premier, fortement attractif, qui inhibe probablement les ovaires; mais nous ne savons pas s'il inhibe la construction des cellules royales (et ce denxième corps est lui-même complexe). Il est vrai que le test de BUTLER le met dans l'impossibilité de rechercher une attractivité éventuelle, puisque de toute façon les abeilles iront lécher le cadavre enduit de miel de leur congénère.

b) La réaction d'inhibition des cellules royales tout au moins dans le test de Vuinitaume, n'est pas spécifique ; on ne peut la déclencher avec des résines banales comme la colophane, mais on y arrive très bien avec la propolis, ou même avec l'extrait alcoolique de bourgeons de peuplier (qui présente avec la propolis toutes sortes de rapports).

\section{Action attractive.}

Test de Pain. - Les abeilles du même âge, écloses à l'étuve depuis 48 heures sont élevées dans une cagette type Liebefeld. On leur présente sur le plancher de la cagette des solutions aux concentrations connues de phérormone.Les abeilles s'abattent sur le papier et y restent en cher- 
chant à le lécher, et en ventilant parfois. On compte toutes les 30 secondes pendant 5 minutes le nombre d'abeilles sur le papier et on en fait la moyenne.

\section{Action sur le développement ovarien.}

Test de Pain. - 55 à 60 jeunes abeilles écloses à l'étuve sont introduites dans une cagette du Liebefeld, nourries avec du candi et du pollen, les deux substances étant présentées séparément. Le pollen est mélangé d'un peu de miel. On fournit de l'eau comme boisson. Les substances à essayer (du type de la phérormone) sont déposées sur des bandes de papier auprès des cupules à pollen dans la cagette. Les abeilles sont disséquées au bout de ro jours et leur degré de développement ovarien évalué suivant la technique de Hzss modifiée par PAIN (I959). Il suit de là que seules les substances attractives peuvent provoquer une inhibition ovarienne, car il faut que les abeilles s'intéressent aux bandes de papier ; or, rien ne les y attire, sauf précisément la phérormone.

\section{Action sur la construction.}

Test de Darchen. - 50 (ou mieux Ioo) jeunes abeilles sont introduites dans une cage d'un volume double de celles du Liebefeld, sans que l'on y place comme d'habitude un morceau de cire ou de rayon. On les nourrit abondamment au miel et on mesure par pesée la quantité de cire produite par les abeilles et façonnée en rayons (ne pas confondre avec les écailles de cire non façonnées qui peuvent tomber de l'abdomen, ni avec l'étirage de la cire gaufrée; il s'agit ici de construction véritable, avec réalisation d'un rayon plus ou moins développé). La surface bâtie est nulle dans ces conditions tant qu'une reine ne se trouve pas dans la cage ; elle est assez grande en présence d'une reine morte posée sur le plancher de la cage ; ou encore si l'on a placé dans la cagette 200 ouvrières pondeuses (orphelines depuis longtemps).

J'ajouterai que cette liste de tests n'est nullement exhaustive. I1 est sûr que la reine ou sa phérormone exercent sur l'ensemble des ouvrières bien d'autres influences que nous ne savons pas encore mesurer.

\section{Localisation de la phérormone.}

Depuis très peu de temps et grâce aux recherches de BUTLER nous savons que la phérormone est produite ou entreposée dans la glande mandibulaire des reines. Elle est répandue de là sur le tégument, soit du fait que la reine se lèche elle-même, soit du fait qu'elle distribue de l'ectohormone aux abeilles et que celles-ci en léchant la reine en régurgitent quelque peu et en enduisent son tégument. De toute façon cette localisa- 
tion nous explique pourquoi la tête des reines avait été trouvée plus active par BUTLER et par PAIN. PAIN a montré d'autre part que la glande mandibulaire des reines attirait très vivement les ouvrières. C'est le seul organe isolé de la reine (à part le tégument) qui soit attractive; la glande mandibulaire d'ouvrière ne l'est pas.

\section{Vature chimique de la phérormone.}

La phérormone est une substance complexe; ses propriétés sont certainement dues à un mélange. Pour l'extraire, PaIN et BARBIER (I960) écrasent des reines au mixer, puis extraient la pâte jusqu'à épuisement par le butanol tertiaire, à une température de $40^{\circ}$. Les extraits filtrés et concentrés sous vide sont repris plusieurs fois par l'acétone chaude. La partie soluble dans l'acétone est ensuite séparee en acides et en neutres par le carbonate de soude. La fraction acide contient le maximum de principes actifs. On soumet cette fraction à la distillation moléculaire et l'on sépare la partie qui passe vers $90^{\circ}$ sous $0,01 \mathrm{~mm}$. Mais cette dernière fraction chromatographiée donne toute une série de zones dont chacune prise isolément est inactive (au test d'attraction de Parn). Seul le mélange de deux et peut-être trois fractions se révèle attractif. L'attractivité résulte donc du mélange de plusieurs fractions déterminées. Le cas est d'ailleurs assez banal en physiologie; et en particulier les spécialistes du comportement n'ignorent pas que si beaucoup de substances pures attirent les insectes, il faut très souvent un mélange pour obtenir le maximum d'effet : ainsi les Moustiques sont attirés vers les Vertébrés à sang chaud par la vapeur d'eau provenant de la transpiration, par le gaz carbonique expiré et aussi par certaines substances odorantes du sang (RAHм, I956). I a cortico-surrénale ne sécrète pas qu'un seul cétostéroïde, mais une trentaine, et leurs fonctions apparaissent dans certains cas comme synergiques, etc.

Pour isoler ce qu'ils appellent "la " phérormone des glandes mandibulaires de la reine (mais nous avons vu que c'est seulement un de ses constituants) BUTLER, CALLOW et JoHnston(I959) épuisent les glandes parl'alcool bouillant pendant quatre heures; le résidu de l'évaporation de l'alcool est repris par l'éther et l'eau et la couche aqueuse extraite par l'éther ; l'extrait éthéré est soumis au partage après évaporation, entre l'éthanol aqueux à $50 \mathrm{p}$. Ioo et l'éther de pétrole. Le produit actif reste dans l'éthanol qu'on lave bien avec l'éther de pétrole; on sèche le résidu de l'éthanol aqueux, on met en solution dans l'éther qu'on lave à l'aide de soude normale. L'extrait alcalin est saturé de gaz carbonique, extrait par l'éther à nouveau, acidulé, repris encore par l'éther. La fraction la plus acide est séparée en deux par chromatographie sur papier (phase stationnaire méthanol aqueux $3 / \mathrm{I}$, phase mobile toluène). La fraction de 
$R_{F} \neq 0,5$ est séparée et éluée par le méthanol. La fraction éthéro soluble de 1'extrait est extraite par un tampon phosphatique à pH 8,3 ; on acidifie ensuite le tampon et on l'épuise par l'éther. On obtient dans ces conditions un corps cristallisé fondant à $45^{-50^{\circ}}$, qui se trouve être un acide $\alpha \beta$ - insaturé avec un groupe carbonyle non conjugué d'un poids moléculaire de I9o, avec trois bandes caractéristiques dans l'infra-rouge (dans KCI) à I709, I689, I643. L'acide décénoïque de la gelée royale d'abord isolé par BUTENANDT et ReMBOLdT (I957) puis par BARKER Foster LAMB et HODGSON (I959) n'a pas de groupe carbonyle non conjugué et présente des bandes dans l'infra-rouge à I 7 Io et $1667 \mathrm{~cm}^{-1}$ notamment. Mais l'acide décénoïque lui-même ou la fraction qui contient son dérivé avec un groupe carbonyle non conjugué ont tous deux été essayés par le test de PAIN et sont dépourvus de pouvoir attractif.

\section{Rapports de la phérormone avec d’autres substances.}

D'après le paragraphe précédent, on a vu que la parenté d'une des fractions constitutives de la phérormone avec l'acide Io-oxy- $\Delta 2$ décénoïque de la gelée royale a été démontrée par BUTLER et ses collaborateurs. Cet acide a été trouvé, pour la première fois dans la nature, par BUTENANDT et REMBOLDT dans la gelée royale. Il ne se rencontre que dans la gelée mais ni dans le nectar ni dans le pollen (dans lequel existe sans doute un de ses précurseurs). Il n'est produit d'après BARkER Foster I,Amb et Hodgson (I959) que dans les glandes mandibulaires de l'ouvrière et ne se tronve pas dans les extraits des autres glandes.

Isolement de l'acide à partir des glandes mandibulaires. - Les glandes reçues dans l'alcool sont épuisées par l'éther. I,es extraits alcooliques et éthérés sont concentrés puis chromatographiés sur papier en présence d'alcool amylique et d'acide formique $5 \mathrm{M}$. A l'aide du rouge de chlorophénol, on met en évidence sur les bandes nue composante acide de $\mathrm{F}_{\mathrm{F}} 0,88$ identique à 1'acide décénoïque. La présence de cet acide est confirmée par ionophorétogramme (suivant́ FosTER, I952) et par ionophorèse.

Double origine de la gelée royale. - On croyait jusqu'ici que la nourriture royale provenait uniquement des giandes pharyngiennes de l'ouvrière, et que par conséquent l'acide décénoïque devait en venir aussi. Il est vrai qu'HAYDAK avait remarqué que le contenu des glandes pharyngiennes, assez limpide, ne prenait l'aspect crémeux caractéristique de la gelée royale que si on le traitait avec la sécrétion des glandes mandibulaires; on sait donc maintenant après les travaux des Anglais que la gelée est le produit de deux types de glandes et non d'un seul.

De plus les glandes mandibulaires de la reine, qui sont, rappelons-le, 
les seules glandes de la reine attractives pour les ouvrières (les glandes homologues des ouvrières ne sont pas attractives pour les ouvrières) modifieraient la molécule d'acide décénoïque et la transformeraient en un des constituants de la phérormone. I a glande mandibulaire de la reine contient d'ailleurs un autre de ces constituants (la substance attractive) et probablement d'autres encore s'il en existe.

\section{L'origine de la phérormone (partie attractive).}

Nous arrivons maintenant à une zone encore fort mal explorée. Si la phérormone I (appelons ainsi la substance de BUTLER, inhibitrice des cellules royales) se trouve dans les glandes mandibulaires, cela n'implique pas du tout que ce soit la reine qui la fabrique. Elle peut se contenter de la transformer, à partir d'un précurseur fourni par les ouvrières qui la nourrissent. PAIN a montré d'ailleurs que la phérormone II (partie attractive de la phérormone totale) peut se développer au bout d'un certain laps de temps chez les reines vierges, mais elle apparaît plus vite et, semble-t-il, à concentration plus élevée lorsque la reine est en contact avec les ouvrières. Pour la phérormone I le précurseur serait évidemment l'acide décénoïque des glandes mandibulaires des ouvrières. Mais ButLeir a montré que la phérormone I elle-même se trouvait dans l'intestin des ouvrières qui ont été en contact, avec la reine et l'ont léchée. Puis PaIn a prouvé avec BARBIER que la phérormone II pouvait être retirée du corps des ouvrières qui ont été en contact avec la reine. Par la même technique qui a servi à extraire la phérormone II de la reine, ces auteurs mettent en évidence dans le corps des ouvrières une quantité de phérormone telle que un peu plus de $70 \mathrm{~g}$ d'abeilles est nécessaire pour en fournir autant qu'une seule reine. Dans ces conditions, un kilog d'abeilles, qui correspond à Io.ooo ouvrières équivaut à I4 reines.

a) Or, d'une part les glandes mandibulaires des ouvrières, nous le répétons, ne sont pas attractives.

b) D'autre part les extraits alcooliques bruts d'ouvrières ne le sont pas davantage, mais le deviennent lorsqu'ils sont purifiés; les extraits alcooliques bruts de reine sont attractifs d'emblée.

c) Enfin, les extraits d'ouvrières orphelines ne donnent, ni avant ni après purification, la moindre trace de phérormone II.

Les hypothèses qu'on peut tirer de ces faits sont assez simples: a) la phérormone II est présente chez l'ouvrière à l'état inactif. La reine se borne peut être à l'activer en l'entreposant dans ses glandes mandibulaires; en même temps et par sa présence elle l'active peut-être aussi chez les ouvrières puisque les ouvrières des ruches orphelines se dispersent plus aisément et ne forment plus la grappe que difficilement : cela pro- 
vient peut-être entre autres causes de ce que leur phérormone ou une partie tout au moins de cette substance n'est plus activée par la reine b) rien ne prouve jusqu'ici que la phérormone des ouvrières vienne; de la reine et une raison peut en faire douter : il existe chez les orphelines un corps qui a des variations très parallèles à celles de la phérormone, bien qu'il ne soit pas attractif. C'est l'antibiotique de LAVIE (I959).

L'antibiotique de Lavie et ses rapports avec la phérormone. - LAVIE a montré dans une série de travaux la présence d'un corps à action antibiotique ou antibactérienne sur le tégument des ouvrières. LAVIE (I959) puis PaIN et LAVIE (I959) ont étudié ses variations dans différentes conditions. Il est très abondant chez l'ouvrière d'âge moyen, mais l'est peu au début et à la fin de la vie des ouvrières. Il existe aussi chez la reine, d'autant mieux qu'elle est restée plus longtemps en contact avec les ouvrières; il est très abondant chez les abeilles orphelines. Or, ce sont les glandes mandibulaires de la reine, qui sont antibiotiques et les autres glandes ne le sont pas. Les glandes mandibulaires des ouvrières ne sont pas antibiotiques, mais les glandes pharyngiennes le sont nettement. De plus, l'antibiotique extrait par l'alcool peut être repris par l'éther; si on lave l'éther avec une solution de carbonate de soude, il passe dans le carbonate; il s'agit donc de corps à fonction acide.

Or la phérormone II se trouve aussi dans la fraction des acides; les glandes mandibulaires des reines ne deviennent fortement attractives que si les ouvrières sont élevées près d'elles et peuvent les nourrir. En un mot les rapports les plus troublants se font voir entre la phérormone et l'antibiotique de LAVIE, avec une seule différence toutefois : 1'antibiotique est particulièrement abondant chez les abeilles élevées en l'absence de reine, alors qu'on n'en peut extraire de phérormone II. Nous n'avons pu jusqu'ici expliquer cette différence ; il y a évidemment deux hypothèses : ou bien la phérormone est réellement absente chez les orphelines ou bien elle se trouve masquée et à l'état de précurseur. Nous penchons vers cette dernière hypothèse. En tout cas, les vieilles abeilles orphelines à très gros ovaires, provenant d'une ruche privée de reine depuis longtemps ne contiennent presque pas d'antibiotique.

\section{Autres substances de la ruche actives sur le comportement.}

\section{Substances d'interattraction ou de familiarisation.}

Elles contribuent toutes à assurer la cohésion du corps social ou à marquer les objets qui " appartiennent " à la colonie. Il en existe un grand nombre que je propose de désigner sous le nom d'épagines (d'ś $\pi \dot{\alpha} \gamma(\omega)$ je place dessus, car il s'agit souvent d'un dépôt de substance). 
Epagine a. - LECOMTE dans ses expériences sur l'interattraction entre abeilles ouvrières a montré que des abeilles dispersées dans une grande cage se regroupent autour d'une cagette contenant déjà un certain nombre de leurs congénères. Dans cette attraction interviennent une composante vibratoire et une composante olfactive qui continue à agir même avec des cadavres d'abeilles toujours attirants pour leurs congénères.

Epagine 3. - En étudiant l'agressivité, LECOMTE remarque que les abeilles placées dans une cagette neuve à l'étuve n'attaquent un leurre agité au milieu d'elles qu'au bout de quelques jours. Mais si avant l'expérience la cagette a été placée vide dans une ruche, de telle manière que les abeilles puissent avoir libre accès à l'intérieur, alors le processus -est accéléré ; les ouvrières introduites par la suite dans la cagette qui a passé dans une ruche un certain "temps de familiarisation " attaquent dès le premier jour le leurre qu'on leur présente. C'est comme si les attaques ne se produisaient que lorsque les ouvrières se "sentent chez elles ", lorsque leur cagette "sent la ruche ". Dans des recherches ultérieures, LECOMTE a montré que l'épagine $\beta$ parait se confondre au moins en partie avec la propolis.

Epagine $\gamma$. - Si les cagettes contenant deux groupes d'abeilles communiquent par une toile métallique ou une tôle perforée dont les orifices permettent les échanges de nourriture, alors les abeilles ne se battent plus quand on enlève la tôle perforée. S'ensuit-il de là,commele veut Ribbands, que l'odeur spéciale du miel contenu dans le jabot (odeur qui ne peut être la même à peu près en aucun cas pour deux colonies, puisqu'elle dépend de la composition florale de l'aire de butinage, qui est excessivement variable pour une même colonie suivant les jours, et à plus forte raison pour deux colonies différentes) soit à 1a base de la reconnaissance entre les abeilles? I1 semble que non : car si l'on distribue dans une des cagettes un miel fortement parfumé avec une essence florale, les bagarres sont vigoureuses lorsqu'on établit la communication avec l'autre cagette, qui a reçu le même miel sans essence; mais elles ne sont pas plus fortes que si les deux cagettes ont reçu le même miel. Comme les échanges de nourriture le prouvent, la reconnaissance doit reposer sur les échanges entre abeilles. Mais nous ne savons ce qui s'échange: -certainement pas le seul contenu du jabot, puisque l'expérience des nourritures très différentes, prouve, contrairement à RiBbands, qu'il ne peut être seul en cause. I ECOMTE songerait plutôt à un complexe issu des glandes salivaires qui aurait un caractère spécial, propre au groupe ; c'est cet ensemble qui constituerait l'épagine $\gamma$.

Quant à comprendre comment deux populations qui viennent de Ia même ruche et qui ont reçu la même nourriture peuvent en arriver à 
différer à ce point en peu de jours, ce n'est pas ici le lieu de l'exposer ; on trouvera une discussion plus approfondie dans LECOMTE (à paraitre).

Epagine $\delta$. - Vuiliaume présente aux abeilles orphelines des cupules de verre oì se trouve déposée une jeune larve avec un peu de gelée royale. Les abeilles l'acceptent très bien. Mais si auprès de ces cupules on leur présente en même temps d'autres cupules que l'on a introduites dans une ruche peuplée vingt-quatre heures avant d'y greffer une jeune larve (il n'est pas nécessaire que les abeilles aient accès aux cuptrles, il suffit qu'elles se trouvent à proximité), alors les cupules ainsi "familiarisées " sont préférées aux cupules neuves : les nourrices les prolongent d'un long tube de cire et y dégorgent de la gelée royale. L'épagine $\delta$ est instable, insoluble dans le benzène, soluble dans l'alcool ; les cupules rincées à l'alcool après la familiarisation ne sont pas mieux acceptées que des cupules neuves.

Epagine \&. - Itorsque les abeilles ont l'habitude de butiner dans une cuptle de verre, on s'aperçoit au bout d'un certain temps que la surface du verre se ternit ; en même temps, le nourrisseur usagé est préféré à un nourrisseur neuf par les abeilles de la ruche; par contre le nourrisseur neuf est préféré au nourrisseur usagé par une ruche étrangère. Si bien que l'épagine $\varepsilon$ joue le rôle de " répulsine " (voir plus loin) pour les étrangères (c'est la "substance attractive et répulsive particulière " suivant la nomenclature de LECOMTE). L'épagine $\varepsilon$ s'extrait par l'alcool des nourrisseurs usagés ou du corps même des butineuses; en ajoutant de l'eau à l'alcool, on la fait facilement passer en phase éthérée.

Epagine క. - C'est la substance de la glande de Nasanov qui attire vivement les abeilles de n'importe quelle ruche (substance attractive générale suivant la nomenclature de I ECOM'TE) lorsqu' on la prélève avec un petit tampon d'ouate à l'orifice même de la glande (technique de RENNER).

Epagine $\eta$. - LECONTE (I956) a observé que les butineuses d'une ruche ayant subi une rotation de $180^{\circ}$ continuent dans une certaine mesure à atterrir à l'ancien emplacement et gagnent le trou de vol à la marche; elles suivent le long de la ruche une piste très étroite, qui est enduite d'une certaine substance entraînable par l'eau, l'alcool ou l'éther : un lavage de la piste à l'aide d'un de ces solvants arrête la procession des abeilles en IO-I5 minutes, et elles se dirigent alors sans coup férir vers la nouvelle position du trou de vol.

Rapports possibles de ces substances entre elles. - Par l'essai au test de VUILLAUME, on peut déjà prouver que les substances $\zeta$ et $\varepsilon$ sont différentes de $\delta$ : elles ne peuvent "familiariser " des cupules de verre 
neuves; mais $\zeta$ diffère aussi d's. Quant à la substance $\beta$ (propolis ou contenue dans la propolis) elle inhibe comme nous le verrons la construction des cellules royales et diffère par conséquent radicalement de $\delta$.

\section{Les substances inhibantes ou répulsives.}

Comme il semble en exister un grand nombre, il vaut mieux les désigner sous le nom général de "répulsines" (de repellere).

Répulsine $\alpha$. - On pourrait ranger sous ce nom la phérormone I qui bloque la construction des cellules royales at test de Butler, mais aussi au test de Vuiliaume. Mais elle n'arrête pas l'étirage des cellules d'ouvrières.

Répulsine $\beta$. - Elle ne serait autre que la propolis qui bloque à la fois et les cellules royales et l'étirage des cellules d'ouvrières (VuILIAUUME). Dans la propolis se trouve aussi nous l'avons vu, l'épagine $\beta$. Nous ignorons le degré de parenté de ces deux substances, n'ayant encore procédé à aucun fractionnement approfondi. Nous savons seulement que la répulsine $\beta$ est entraînée par la vapeur d'eau quand on chauffe la propolis dans l'eau alcalinisée par to p. roo de soude; mais elle ne se trouve pas dans l'essence de propolis qui distille sans alcalinisation préalable. Une substance similaire se rencontre enfin dans les bourgeons du peuplier, ce qui laisse supposer pour cette répulsine une origine exogène.

Répulsine $\gamma$. - Elle est contenue dans le venin, qui excite beaucoup les attaques entre ouvrières de deux cagettes lorsqu'on fait circuler de l'air ayant barboté dans une dissolution de venin.

Répulsine $\delta$. - Elle n'est autre que l'épagine $\varepsilon$ agissant sur une ruche étrangère.

Répulsine $\varepsilon$. - A l'épagine $\varepsilon$ se trouve normalement mélangée une autre substance répulsive pour les abeilles de la même ruche, mais dont l'effet est normalement surpassé de loin par l'épagine associée. Toutefois lorsqu'on fait un partage entre phase hydro-alcoolique et phase éthérée, l'éther enlève la substance de familiarisation et il ne reste dans l'alcool qu'un corps répulsif pour les abeilles de la propre ruche d'oì on l'a extrait (substance répulsive particulière de LECOMTE).

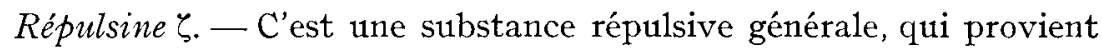
de l'épuisement par l'alcool du corps des abeilles non plus entières, mais coupées en morceaux ou broyées. La dilacération des tissus produit chez l'abeille comme peut-être chez les Vairons étudiés par von Frisch, une substance qui éloigne tous les congénères, à quelque ruche qu'elles appartiennent. 
Les substances de butinage. (Allectines $d u$ latin allicio).

Je ne veux que les mentionner très brièvement, d'abord parce qu'on ne sait rien de leur nature, ensuite parce que leur action s'exerce en dehors de la ruche. Notons tout de même que les abeilles sont attirées. très vivement par le miel, par le pollen (ici LouvEAux a commencé des fractionnements qui tendraient à prouver que la substance attractive se trouve dans la fraction stérolique) vers la propolis, qu'on peut parfaitement leur faire butiner dans une assiette, et même vers l'eau sale (on sait ici que les traces d'indol et de scatol que contiennent les eaux polluées sont les agents attractifs).

\section{Conclusion.}

Il est bien évident que la liste de substances actives que je viens d'énumérer n'est nullement limitative. Il nous paraît probable, à la lueur de ce que nous savons déjà qu'une très grande partie des activités de la ruche doit être réglée par des échanges de substances (la nourriture ne constitue qu'une partie des échanges). Mais dans un domaine sans doute immense, nous venons à peine de pénétrer.

\section{RÉFÉRENCES BIBLIOGRAPHIQUES}

Barker (S. A.), Foster (A. B.), Lamb (D. C.), Jackman (L. M.). - Biological origin and configuration of Io-hydroxy- $\Delta_{2}$ decenoic acid. Nature, 184, 634, I959.

Barker (S. A.), Foster (A. B.), Lamb (D. C.), Hodgson (N.). - Identification of Io-Hydroxy- $\Delta_{2}$ decenoic acid in royal jelly. Nature, 183, 996-7, I959.

ButENANDT (A.), REMBOLd (H.). - Uber den Weiselzellenfuttersaft der Honigbiene. I. Isolierung, Konstitutionsermittlung und Vorkommen der Io-Hydroxy- $\lrcorner_{2}$ decensäure. Hoppe Seyler's $Z$. Physiol. Chem., 308, 284-9, I957.

Butrer (C. G.). - Trans. Roy. Entom. Soc., 105, II, I954.

Butler (C. G.). - Proc. Roy. Entom. Soc., A, 31, I2, I954.

BUtler (C. G.), Callow (R. K.), Johnston (N. C.). - Extraction and purification of queen substance from queen bees. Nature, 4702, I87I, I959.

Butlez (C. G.), Gibbons (D.). - J. Ins. Physiol., 2, 6r, I958.

Darchen (R.). - Les techniques de construction chez Apis mellifica. Ann. Sc. Nat. I $2^{\mathrm{e}}$ série, I, II3-209, I959.

HEsS (H.). - Uber den Einfluss der Weisellösigkeit und des Fruchtbarkeitsvitamins E auf der Ovarien der Bienenarbeiterin. Beih. Schreiz. Bienenz., Jan. I942.

Haydak (M.). - - Bee World, 38, I97, I957.

Karson (P.), Pheromones Ergeb. Biol. 22, 212-25, 1960.

Lavie (P.). - Etude des substances antibiotiques présentes chez Apis mellifica et chez quelques Insectes sociaux. C. R. Acad. Sc., 244, 2653-55, I 957

LAviE (P.). - Action antibiotique de quelques substances provenant de l'Abeille ou de la ruche sur Bacillus larvae et Bacillus alvei. C. R. Acad. Sc., 248, 455-7, I959. 
LAvie (P.), PaIN (J.). - Les rapports entre la substance antibiotique des reines et des ouvrières d'abeilles, le développement ovarien et l'ectohormone. C. R. Acad. Sc., 248, I587-89, I959.

Lavie (P.), PaIN (J.). - Relation entre la substance attractive, le facteur antibiotique et le développement ovarien chez la reine d'Abeille. C.R. Acad. Sc., 248, 3753-55, I959.

LECOMTE (J.). - Recherches sur le comportement agressif des ouvrières d'Apis mellifica. Behaviour, IV, 6o-6, I95I.

LECOMTE (J.). - Hétérogénéité dans le comportement agressif des ouvrières d'A pis mellifica. C. R. Acad. Sc., 234, 890-I, I952.

LECOMTE (J.). - Formation de pistes par les butineuses d'une ruche ayant subi une rotation de $180^{\circ} . Z$. Bienenforsch., 3, H. 6 , I956.

PaIN (J.). - Sur l'ectohormone des reines d'Abeilles. C. R. Acad. Sc., 239, I869-70, r954.

PAIN (J.). - Influence des reines mortes sur le développement ovarien de jeunes ouvrières d'A pis mellifica. Ins. Soc., 11, 35-43, I955.

PaIn (J.). - Étude de 1'apparition de 1'attractivité chez les reines vierges d'Abeille. C. R. Acad. Sc., 248, 32I I-I2, I959.

Pain (J.), Barbier (M.). - Mise en évidence d'une substance attractive extraite du corps des ouvrières d'Abeilles non orphelines. C. R. Acad. Sc. (en cours de publication), Ig60.

Rahm (U.). - Zum Problem von Stechmücken durch den Menschen. Acta tropica, 13, 3I9-44, I956.

Vuillaume (M.). - Contribution à la psychophysiologie de l'élevage des reines chez les Abeilles. Ins. Soc., 2, II3-57, I957.

VuillaUme (M.). - Les substances inhibitrices de la construction des cellules royales chez les Abeilles. C. $R$. Acad. Sc., 246, I298-9, I958.

Vuiliaume (M.). - Nouvelles données sur la psychophysiologie de l'élevage des reines chez Apis mellifica. Ann. Abeille, 11, II3-38, I959. 